\title{
Superkicks in Hyperbolic Encounters of Binary Black Holes
}

\author{
James Healy, ${ }^{1}$ Frank Herrmann, ${ }^{1}$ Ian Hinder, ${ }^{1}$ Deirdre M. Shoemaker, ${ }^{1,2}$ Pablo Laguna, ${ }^{1,2}$ and Richard A. Matzner ${ }^{3}$ \\ ${ }^{1}$ Center for Gravitational Wave Physics \\ The Pennsylvania State University, University Park, PA 16802 \\ ${ }^{2}$ Center for Relativistic Astrophysics and School of Physics \\ Georgia Institute of Technology, Atlanta, GA 30332 \\ ${ }^{3}$ Center for Relativity and Department of Physics \\ The University of Texas at Austin, Austin, TX 78712
}

\begin{abstract}
Generic inspirals and mergers of binary black holes produce beamed emission of gravitational radiation that can lead to a gravitational recoil or kick of the final black hole. The kick velocity depends on the mass ratio and spins of the binary as well as on the dynamics of the binary configuration. Studies have focused so far on the most astrophysically relevant configuration of quasi-circular inspirals, for which kicks as large as $\sim 3,300 \mathrm{~km} \mathrm{~s}^{-1}$ have been found. We present the first study of gravitational recoil in hyperbolic encounters. Contrary to quasi-circular configurations, in which the beamed radiation tends to average during the inspiral, radiation from hyperbolic encounters is plunge dominated, resulting in an enhancement of preferential beaming. As a consequence, it is possible to achieve kick velocities as large as $10,000 \mathrm{~km} \mathrm{~s}^{-1}$.
\end{abstract}

PACS numbers: 04.60.Kz,04.60.Pp,98.80.Qc

Numerical relativity estimates of the gravitational recoil or kick inflicted on the final black hole $(\mathrm{BH})$ from generic inspirals and mergers of binary black holes $(\mathrm{BBH})$ have triggered tremendous excitement in astrophysics. This is mainly due to the fact that most galaxies host a supermassive black hole (SMBH) at their centers [1, 2]. As galaxies merge, a kick to the final $\mathrm{BH}$ from the coalescence of the $\mathrm{BHs}$ at the galactic cores could have profound implications in subsequent mergers, affecting the growth of SMBHs via mergers as well as the population of galaxies containing SMBHs. In addition, there have been several suggestions of direct observational signatures of putative BH recoils [3, 4, [5, 6, 7, , 8], with one study [9] presenting evidence for the first candidate of a recoiling SMBH.

BH kick velocities depend on the mass ratio and spins of the merging BHs as well as on the initial configuration and subsequent dynamics of the binary. Studies published to date have concerned the most astrophysically relevant configuration, that of quasi-circular inspirals 10, 11, 12, 13]. One remarkable discovery has been kicks of a few thousand $\mathrm{km} \mathrm{s}^{-1}$ found in configurations of equal-mass binaries with initially anti-aligned spins in the orbital plane [14, 15, 16]. For near extremal spins $(a / m=0.925)$, recoils as large as $3,300 \mathrm{~km} \mathrm{~s}^{-1}$ have been computed [17].

Motivated by our previous study [18] of the final spin of $\mathrm{BHs}$ from scattering mergers of $\mathrm{BBHs}$, we present the first extension of gravitational recoil to hyperbolic encounters. There are crucial differences between hyperbolic and quasi-circular configurations that affect the kick to the final BH. For quasi-circular orbits, the emitted radiation is asymmetrically beamed, and carries linear momentum with it. But it tends to average out during the inspiral [19], producing a modest wobbling and drift of the center of mass of the binary. The final kick arises because as the binary approaches the plunge, the averaging loses its effectiveness, leading to a gradual recoil build-up. Both numerical simulations and postNewtonian studies 19, 20] have confirmed the gradual kick accumulation during the inspiral, and, in addition, the studies have shown that most of the recoil is generated during the plunge. In some instances, during the plunge and ring-down there is also a period of anti-kick before reaching the final kick value [21, 22].

The main motivation for the present study was to consider plunge-dominated configurations to investigate whether kicks comparable to those for quasi-circular inspirals can be found. We were surprised to find that kicks larger than $3000 \mathrm{~km} \mathrm{~s}^{-1}$ are in general produced for spin configurations equivalent to those studied in quasicircular inspirals. We show below a configuration giving kick velocities as large as $10,000 \mathrm{~km} \mathrm{~s}^{-1}$. Two qualitative features of hyperbolic encounters contribute to these larger kicks. Not only are hyperbolic encounters plunge dominated, but the nature of the plunge is such that it enhances the beaming of radiated linear momentum [23].

We use the same computational infrastructure and methodology as in previous studies [10, 12, 18], namely a BSSN code with moving puncture gauge conditions. The hyperbolic encounters are initiated with Bowen-York initial data [24]. The data consist of two equal-mass BHs with masses $m=M / 2$ located along the $x$-axis: $\mathrm{BH}_{ \pm}$is located at $x= \pm 5 M$ and has linear momentum $\vec{P}_{ \pm}= \pm(P \cos \theta, P \sin \theta, 0)$ with $\theta$ the angle in the orbital plane with respect to the $x$-axis. The total initial orbital angular momentum is then $\vec{L} / M^{2}=10(P / M) \sin \theta \hat{z}$. The spins of each $\mathrm{BH}$ are in the orbital plane: $\mathrm{BH}_{ \pm}$ has spin $\vec{S}_{ \pm}= \pm\left(S \cos \phi_{ \pm}, S \sin \phi_{ \pm}, 0\right)$, with $\phi_{ \pm}$the angle in the orbital plane with respect to the $\pm x$-axis. The parameter space of our encounters is quite large: $\left\{P, \theta, S, \phi_{ \pm}\right\}$. However, from exploratory runs we have gained a good understanding of the parameter space and isolated those parameters that can be kept fixed without 


\begin{tabular}{cccrrrr}
\hline \hline$P / M$ & $\phi_{+}$ & $L / M^{2}$ & $V_{\|}$ & $V_{\perp}$ & $V$ & $E_{\mathrm{rad}}(\%)$ \\
\hline 0.2379 & 0 & 1.064 & 5155.2 & -0.1 & 5155.2 & 4.9 \\
0.2665 & 0 & 1.192 & 6561.0 & -0.2 & 6561.0 & 7.6 \\
0.2739 & 0 & 1.225 & 6505.3 & -0.3 & 6505.3 & 8.4 \\
0.2851 & 0 & 1.275 & 5424.5 & -0.3 & 5424.5 & 10.1 \\
0.2952 & 0 & 1.320 & 3140.3 & -0.4 & 3140.3 & 11.8 \\
\hline 0.2379 & 45 & 1.064 & 5483.9 & 709.7 & 5529.6 & 5.2 \\
0.2665 & 45 & 1.192 & 7614.9 & 932.4 & 7671.8 & 8.0 \\
0.2739 & 45 & 1.225 & 7830.2 & 930.5 & 7885.3 & 8.9 \\
0.2851 & 45 & 1.275 & 7227.6 & 778.5 & 7269.4 & 10.4 \\
0.2952 & 45 & 1.320 & 5026.4 & 384.7 & 5041.0 & 12.2 \\
\hline 0.2379 & 90 & 1.064 & 4291.4 & 1074.6 & 4423.9 & 5.5 \\
0.2665 & 90 & 1.192 & 6485.7 & 1519.7 & 6661.3 & 8.7 \\
0.2739 & 90 & 1.225 & 6740.7 & 1528.0 & 6911.6 & 9.6 \\
0.2851 & 90 & 1.275 & 6001.4 & 1197.7 & 6119.7 & 11.5 \\
0.2952 & 90 & 1.320 & 3791.3 & 410.6 & 3813.3 & 13.1 \\
\hline \hline
\end{tabular}

TABLE I: Configuration parameters $P / M, \phi_{+}$and initial orbital angular momentum $L / M^{2}$ for the cases with $\phi_{-}=0^{\circ}$ and $S / M^{2}=0.2$. The last four columns show the corresponding kick velocities of the final $\mathrm{BH}$ in $\mathrm{km} \mathrm{s}^{-1}$ as well as the $\%$ of energy radiated.

seriously compromising the goals of the study.

For most of the runs, we have kept the spin magnitudes at $S / M^{2}=0.2(a / m=0.8)$, with the exception of those runs used to investigate the dependence of the kick on $S$. We kept fixed also the impact angle at $\theta=153.4^{\circ}$. We considered some other angles but found that this is the angle for which we obtained the largest kicks. Finally, for most cases we kept the spin direction of $\mathrm{BH}_{-}$located at $x=-5 \mathrm{M}$ fixed at $\phi_{-}=0^{\circ}$ or $45^{\circ}$. Once other parameters were fixed from among a small set of values, the parameter we varied in general was the linear momentum magnitude $P$.

Kicks are computed from a surface integral [25, 26] involving the Weyl curvature tensor $\Psi_{4}$. Although strictly speaking this kick formula must be evaluated in the limit $r \rightarrow \infty$, we applied it at extraction radii $r / M=$ $\{40,50,75,85,100\}$. The resultant kicks were fitted to both $V(r)=V_{\infty}^{(1)}+K_{0} / r$ and $V(r)=V_{\infty}^{(2)}+K_{1} / r+$ $K_{2} / r^{2}$. The extrapolated $r \rightarrow \infty$ kicks and their errors were estimated from $V_{\infty}=\left(V_{\infty}^{(1)}+V_{\infty}^{(2)}\right) / 2$ and $\delta V_{\infty}=\left|V_{\infty}^{(1)}-V_{\infty}^{(2)}\right|$, respectively. Unless explicitly noted, all the reported kick velocities and energy radiated were obtained from simulations with resolution $h=M / 0.8$ on the mesh used for the kick computation. Every run had 10 levels of factor-of- 2 refinement, with outer boundaries at $\sim 320 \mathrm{M}$. We discuss below the convergence of kick estimates as the grid spacing is decreased and the extraction radius is increased. The estimated errors in all the kick results presented here due to the finite differencing grid spacing $h=M / 0.8$ and extrapolation $r \rightarrow \infty$ are of the order of a few hundred $\mathrm{km} \mathrm{s}^{-1}$.

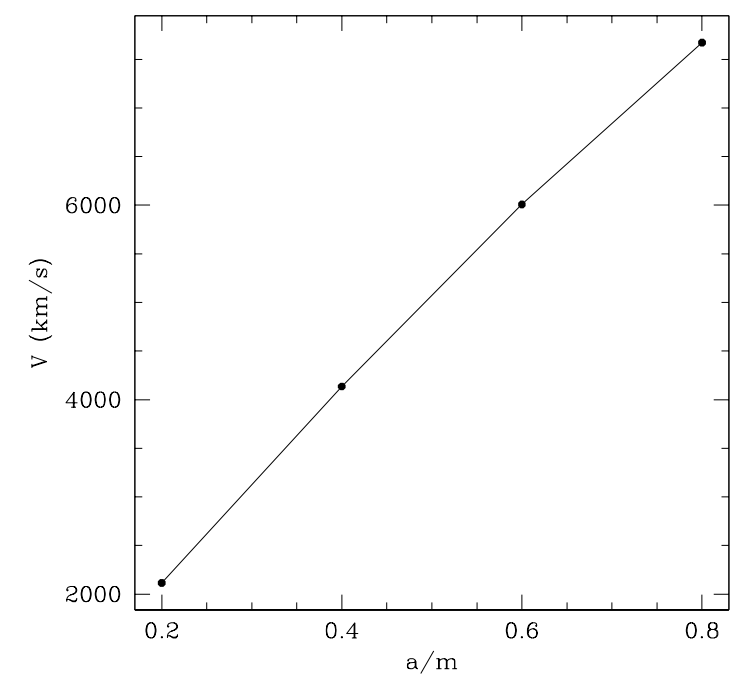

FIG. 1: Magnitude of the kick velocity as a function of the magnitude of the initial $\mathrm{BH}$ spin $(a / m)$ for the case $(P / M=$ $0.2665, \phi_{+}=45^{\circ}$ )

Table I gives the components of the recoil along the initial orbital angular momentum $V_{\|}$and in the initial orbital plane $V_{\perp}$ in $\mathrm{km} \mathrm{s}^{-1}$ (and the total recoil $V$ ) for the cases with $\phi_{-}=0^{\circ}$. Notice that the largest recoil in this case happens when $\phi_{+}=45^{\circ}$. Another important observation is that, although the dominant component of the kick is $V_{\|}$, as we increase $\phi_{+}$a substantial component of the kick is also generated in the orbital plane (see $V_{\perp}$ ). The rightmost column in Table【 gives the $r \rightarrow \infty$ extrapolated radiated energy as a percentage of the total initial energy of the binary. We see that large angular momentum increases the energy radiated, up to very substantial values, as large as $13 \%$. For even larger angular momenta, we expect a falloff of the radiated energy.

For the case $P / M=0.2665, \phi_{+}=45^{\circ}$ in Table 【 we have carried out simulations to investigate the dependence of the kick with the initial BH spins $(a / m)$. The results are displayed in Fig. 1. We find that, to first order, the kick is proportional to $a / M_{h}$, as found in quasicircular orbits [12]. However, as the initial spin of the BHs grows, we found hints of the quadratic spin dependence also obtained in quasi-circular orbits [27].

The configuration we have found to yield the largest kick has the spins anti-aligned $\phi_{+}=\phi_{-}=\phi$, as in the case of quasi-circular orbits [14, 15, 16], and initial linear momentum $P / M=0.3075$, corresponding to initial angular momentum $L / M^{2}=1.275$. The kicks are essentially along the direction of the initial orbital angular momentum. Figure 2 shows the kick velocity $V_{\|}$as a func- 


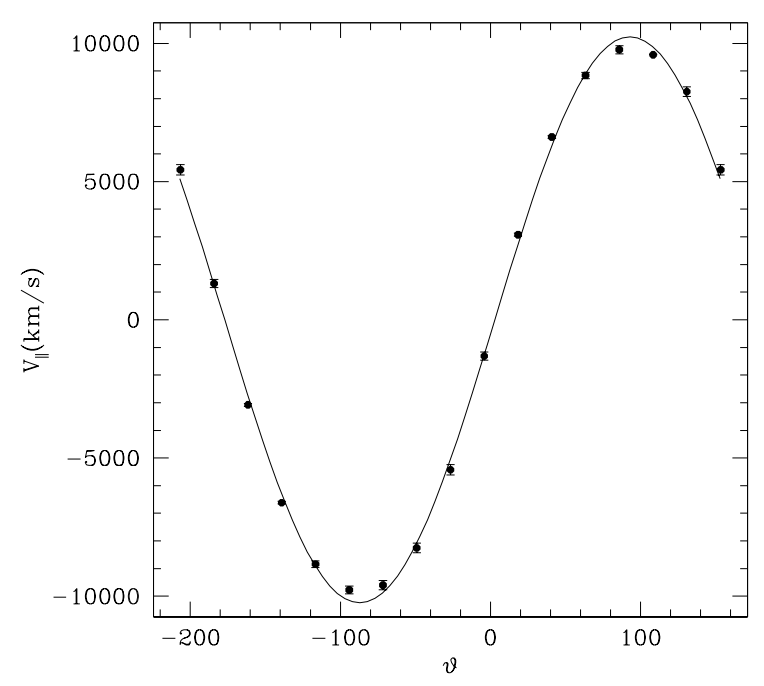

FIG. 2: Kick velocity $V_{\|}$as a function of $\vartheta=\theta-\phi$ for the cases with spin orientation $\phi_{+}=\phi_{-}=\phi$ and initial linear momentum $P / M=0.3075$, corresponding to initial angular momentum $L / M^{2}=1.275$. The solid line represents the fit $V_{\|}=-10,256 \cos \left(\vartheta+86^{\circ}\right) \mathrm{km} \mathrm{s}^{-1}$.

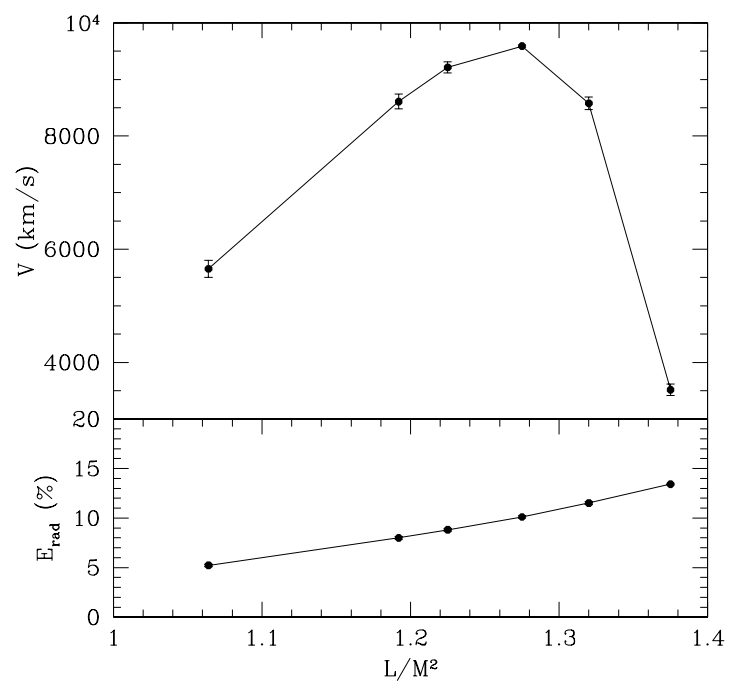

FIG. 3: Magnitude of the kick velocity (top panel) and \% of energy radiated (bottom panel) as a function of initial orbital angular momentum for spin orientation $\phi_{+}=\phi_{-}=45^{\circ}$.

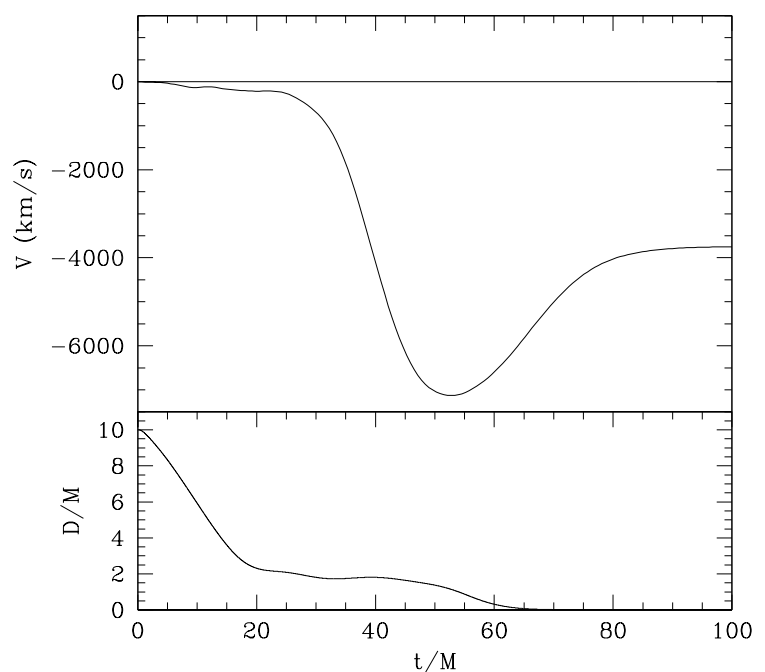

FIG. 4: Accumulation of the kick velocity extracted at $r=$ $75 M$ (top panel) and binary separation (bottom panel) as a function of time for the case $L / M^{2}=1.375, \phi_{+}=\phi_{+}=45^{\circ}$ in Fig. 3

tion of $\vartheta=\theta-\phi$, where $\vartheta$ measures the angle between the initial spin and linear momentum vectors. The solid line represents the fit $V_{\|}=-10,239 \cos \left(\vartheta+86^{\circ}\right) \mathrm{km} \mathrm{s}^{-1}$. This is the same angular dependence found in quasicircular orbits [16]. Furthermore, as with circular inspirals, the maximum kick is obtained for $\vartheta \approx 90^{\circ}$ although in these hyperbolic encounters the kick is significantly larger, close to $10,000 \mathrm{~km} \mathrm{~s}^{-1}$.

To investigate the dependence on the initial orbital angular momentum, we selected the case $\phi_{+}=\phi_{-}=\phi=$ $45^{\circ}$ or $\vartheta=108^{\circ}$ in Fig. 2 which yields a kick magnitude of $9,589 \mathrm{~km} \mathrm{~s}^{-1}$ and $15 \%$ energy radiated, and carried out simulations varying the initial momentum of the BHs. Figure 3 shows the magnitude of the kick velocity $V$ (top panel) and the energy radiated $E_{\text {rad }}$ in $\%$ of the initial energy (bottom panel) as a function of $L / M^{2}$. Note that the maximum of $V$ does not occur for maximum $E_{\text {rad }}$. The case with the largest initial angular momentum, $L / M^{2}=1.375$, has an interesting feature as displayed in the top panel of Fig. 4. There is a pronounced anti-kick before the recoil reaches its final value. The reason for this anti-kick could be due to the fact that the plunge is not as pronounced, appearing more circularlike. That is, there is a decrease in the rate at which the binary comes together, as one can see in the bottom panel of Fig. 4 during the time interval 20-40 $M$. Thus, the flux vectors responsible for the kick have more time to undergo the phase shift needed for the appearance of 


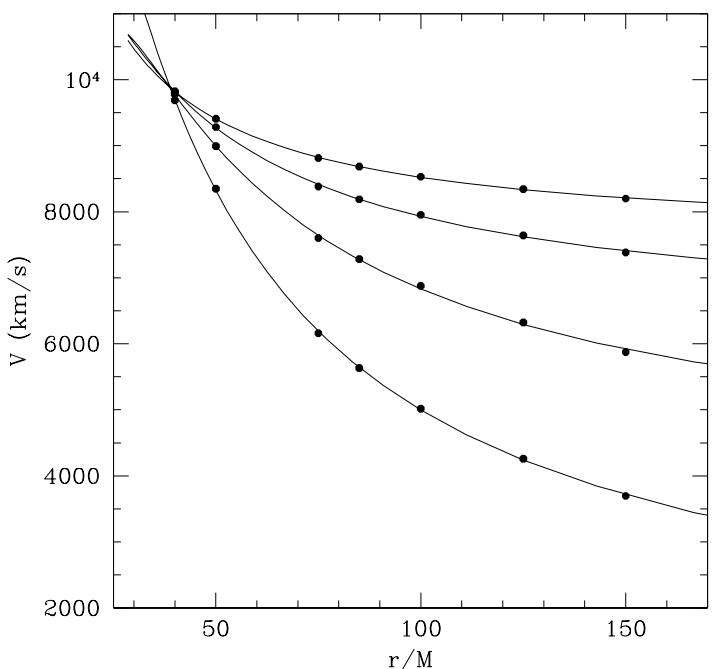

FIG. 5: Magnitude of the kick velocity as a function of the extraction radius for different resolutions in the case $\left(P / M=0.2665, \phi_{+}=45^{\circ}\right)$. From bottom to top are respectively resolutions of $h=\{M / 0.4, M / 0.5, M / 0.63, M / 0.8\}$ at the extraction level. Dots are the data and lines correspond to the fit $V(r)=V_{\infty}^{(2)}+\left(K_{1} / r+K_{2} / r^{2}\right)$.

an anti-kick 22].

In addition to the errors from extracting the gravitational recoil at a finite radius, the values of the kicks are affected by numerical finite differencing resolution. To investigate these errors, we selected the case $P / M=0.2665, \phi_{+}=45^{\circ}$ from Table II and carried out simulations with resolutions $h=$ $\{M / 0.4, M / 0.5, M / 0.63, M / 0.8\}$ on the extraction level.
Figure 5 shows the data (points) and the corresponding fitting (lines) to $V(r)=V_{\infty}^{(2)}+K_{1} / r+K_{2} / r^{2}$. We find that at a given extraction radius, the error in the kick scales as $h^{3}$, and the kick itself grows with resolution. Based on this $h^{3}$ behavior, we estimate the error in the kick extrapolated as $r \rightarrow \infty$ computed using $h=M / 0.8$ to be of the order of a few hundred $\mathrm{km} \mathrm{s}^{-1}$, and we expect all the kicks presented to be of a similar accuracy.

We have carried out a study of the gravitational recoil of the final $\mathrm{BH}$ in the merger of hyperbolic $\mathrm{BBH}$ encounters. We have found that in general the kick velocities for in-plane initial BH spins are significantly larger than those from the corresponding quasi-circular mergers. Our results suggest that kicks as large as $10,000 \mathrm{~km} \mathrm{~s}^{-1}$ are possible. We have also found that the dependence of the kick on the initial magnitude of the BH's spins is similar to the quasi-circular case. An analytic multipolar analysis of encounters for similar configurations can be found in Ref. 23]. A recent study by O'Leary, Kocsis and Loeb 28] has found that in dense population environments, there is a non-negligible probability for close flybys or hyperbolic encounters [29]. Most of the cases they considered are those in which after the first passage, the BHs release enough energy to become bound with large initial eccentricity. The hyperbolic encounters we consider in the present work are the extreme case of immediate merger. Nonetheless, kicks with the magnitudes found in the present study could lead to interesting astrophysical consequences.

Work supported in part by NSF grants PHY-0653443 (DS), PHY-0555436 (PL), PHY-0653303 (PL,DS), PHY0114375 (CGWP), PHY-0354842 (RM) and NASA grant NNG-04GL37G (RM). Computations carried out under LRAC allocation MCA08X009 (PL,DS) and at the Texas Advanced Computation Center, University of Texas at Austin. We thank M. Ansorg, T. Bode, A. Knapp and E. Schnetter for contributions to our computational infrastructure.
[1] D. Richstone, E. A. Ajhar, R. Bender, G. Bower, A. Dressler, S. M. Faber, A. V. Filippenko, K. Gebhardt, R. Green, L. C. Ho, et al., Nature (London) 395, A14+ (1998), arXiv:astro-ph/9810378.

[2] R. Decarli, G. Gavazzi, I. Arosio, L. Cortese, A. Boselli, C. Bonfanti, and M. Colpi, MNRAS 381, 136 (2007), arXiv:0707.0999.

[3] G. A. Shields and E. W. Bonning, ArXiv e-prints 802 (2008), 0802.3873.

[4] B. Devecchi, M. Dotti, E. Rasia, M. Volonteri, and M. Colpi, ArXiv e-prints 805 (2008), 0805.2609.

[5] L. Blecha and A. Loeb, ArXiv e-prints 805 (2008), 0805.1420.

[6] Z. Lippai, Z. Frei, and Z. Haiman, Astrophys. J. Lett. 676, L5 (2008), arXiv:0801.0739.

[7] A. Loeb, Physical Review Letters 99, 041103 (2007),
arXiv:astro-ph/0703722.

[8] B. Kocsis and A. Loeb, ArXiv e-prints 803 (2008), 0803.0003.

[9] S. Komossa, H. Zhou, and H. Lu, Astrophys. J. Lett. 678, L81 (2008), arXiv:0804.4585.

[10] F. Herrmann, I. Hinder, D. Shoemaker, and P. Laguna, Classical and Quantum Gravity 24, 33 (2007), arXiv:grqc/0601026.

[11] J. A. González, U. Sperhake, B. Brügmann, M. Hannam, and S. Husa, Physical Review Letters 98, 091101 (2007), arXiv:gr-qc/0610154.

[12] F. Herrmann, I. Hinder, D. Shoemaker, P. Laguna, and R. A. Matzner, Astrophys. J. 661, 430 (2007), arXiv:grqc/0701143.

[13] M. Koppitz, D. Pollney, C. Reisswig, L. Rezzolla, J. Thornburg, P. Diener, and E. Schnetter, Physical Re- 
view Letters 99, 041102 (2007), arXiv:gr-qc/0701163.

[14] J. A. Gonzalez, M. D. Hannam, U. Sperhake, B. Brugmann, and S. Husa, Phys. Rev. Lett. 98, 231101 (2007), gr-qc/0702052.

[15] M. Campanelli, C. Lousto, Y. Zlochower, and D. Merritt, Astrophys. J. Lett. 659, L5 (2007), arXiv:gr-qc/0701164.

[16] M. Campanelli, C. O. Lousto, Y. Zlochower, and D. Merritt, Physical Review Letters 98, 231102 (2007), arXiv:gr-qc/0702133.

[17] S. Dain, C. O. Lousto, and Y. Zlochower, ArXiv e-prints 803 (2008), 0803.0351.

[18] M. C. Washik, J. Healy, F. Herrmann, I. Hinder, D. M. Shoemaker, P. Laguna, and R. A. Matzner, ArXiv eprints 802 (2008), 0802.2520.

[19] L. Blanchet, M. S. S. Qusailah, and C. M. Will, Astrophys. J. 635, 508 (2005), arXiv:astro-ph/0507692.

[20] T. Damour and A. Gopakumar, Phys. Rev. D 73, 124006 (2006), arXiv:gr-qc/0602117.

[21] J. G. Baker, W. D. Boggs, J. Centrella, B. J. Kelly, S. T. McWilliams, M. C. Miller, and J. R. van Meter, Astrophys. J. 668, 1140 (2007), arXiv:astro-ph/0702390.
[22] J. D. Schnittman, A. Buonanno, J. R. van Meter, J. G. Baker, W. D. Boggs, J. Centrella, B. J. Kelly, and S. T. McWilliams, Phys. Rev. D 77, 044031 (2008), arXiv:0707.0301.

[23] S. H. Miller and R. A. Matzner (2008), 0807.3028.

[24] J. M. Bowen and J. W. York, Phys. Rev. D 21, 2047 (1980).

[25] E. T. Newman and K. P. Tod, General Relativity and Gravitation: One hundread years after the birth of Albert Einstein, vol. 2 (Plenum Press N.Y., 1980).

[26] M. Campanelli and C. O. Lousto, Phys. Rev. D59, 124022 (1999), gr-qc/9811019.

[27] D. Pollney, C. Reisswig, L. Rezzolla, B. Szilágyi, M. Ansorg, B. Deris, P. Diener, E. N. Dorband, M. Koppitz, A. Nagar, et al., Phys. Rev. D 76, 124002 (2007), arXiv:0707.2559.

[28] R. M. O'Leary, B. Kocsis, and A. Loeb, ArXiv e-prints 807 (2008), 0807.2638.

[29] B. Kocsis, M. E. Gaspar, and S. Marka, Astrophys. J. 648, 411 (2006), astro-ph/0603441. 